Journal of

Synchrotron

Radiation

ISSN 0909-0495

Received 13 January 2004

Accepted 18 November 2004

\section{Specimen charging in X-ray absorption spectroscopy: correction of total electron yield data from stabilized zirconia in the energy range 250-915 eV}

\author{
Dimitrios Vlachos, $^{\mathrm{a}, \mathrm{b}} \neq$ Alan J. Craven ${ }^{\mathrm{a} *}$ and David W. McComb ${ }^{\mathrm{b}}$ 化 \\ ${ }^{\mathbf{a}}$ Department of Physics and Astronomy, University of Glasgow, Glasgow, UK, and ${ }^{\mathbf{b}}$ Department of \\ Chemistry, University of Glasgow, Glasgow, UK. E-mail: a.craven@physics.gla.ac.uk
}

\begin{abstract}
The effects of specimen charging on X-ray absorption spectroscopy using total electron yield have been investigated using powder samples of zirconia stabilized by a range of oxides. The stabilized zirconia powder was mixed with graphite to minimize the charging but significant modifications of the intensities of features in the X-ray absorption near-edge fine structure (XANES) still occurred. The time dependence of the charging was measured experimentally using a time scan, and an algorithm was developed to use this measured time dependence to correct the effects of the charging. The algorithm assumes that the system approaches the equilibrium state by an exponential decay. The corrected XANES show improved agreement with the electron energy-loss near-edge fine structure obtained from the same samples.
\end{abstract}

Keywords: X-ray absorption near-edge structure; electron energy-loss near-edge structure; charging; correction for charging; total electron yield.
(C) 2005 International Union of Crystallography Printed in Great Britain - all rights reserved probes of the site and symmetry-projected density of unoccupied electronic states around the excited atom.

In general, EELS is restricted to energy losses of less than $\sim 2 \mathrm{keV}$ owing to the rapid decrease of the signal with energy loss. The advantage of the technique is that it can be carried out with sub-nanometre spatial resolution when the spectrometer is coupled to a modern nanoanalytical electron microscope. In contrast, XAS can be used to record the ionization edge from almost any bound electron using the high-intensity $\mathrm{X}$-ray radiation available from a synchrotron facility. However, the limited focusing possible when using X-rays on a typical synchrotron beamline normally means that lateral spatial resolution in XAS is of the order of millimetres.

The simplest and most widely used detection technique in XAS experiments in the soft X-ray region is total electron yield (TEY). The TEY signal consists of all the electrons escaping from the specimen as a result of the cascade process started by the initial absorption of each photon. Close to the threshold of an edge, the contribution of that edge to the TEY signal is dominated by the contribution from the cascade process initiated by the ejected Auger electron as the photoelectron has little energy (Henke et al., 1979). However, the underlying background also has contributions arising from high-energy photoelectrons ejected from much less tightly bound atomic states, the Auger electrons from which can have much lower energy. Although other detection techniques can be used, the TEY method is popular because of the large signal available and the ease of measuring the signal via the 
drain current from the sample. It has been shown that TEY can be used to derive absorption coefficients (Elam et al., 1988; Henke et al., 1981; Owens et al., 1997; Erbil et al., 1988). The main disadvantages of TEY are the range of mechanisms giving rise to escaping electrons and the relatively small depth probed in the sample.

For insulating specimens, there is also the problem of sample charging. There are many papers dealing with how sample charging affects a range of techniques (e.g. Cros, 1992; Cazeau, 1999; Seah \& Spencer, 2000). Charging affects the energies of electrons and hence causes major shifts of features in X-ray photoemission spectroscopy (XPS) and Auger electron spectroscopy (AES). However, in studying XANES, the energy is defined by the incident radiation and so the effect is on the intensity of the signal collected when using TEY (Gilbert et al., 2000). In the cases of ELNES, the energy lost by the primary energy is measured. Provided that the same current is incident on the specimen while the spectrum is recorded, the charge on the specimen is constant. This charge will cause the primary electron to lose a little kinetic energy before reaching the specimen but it will regain the same amount leaving the specimen. Thus neither the energy nor the intensity of a feature in ELNES is affected by equilibrium charging.

A widespread method of avoiding any charging effect at insulating materials is the performance of XAS measurements by TEY detection in the presence of an inert gas atmosphere such as helium. The gas ionization process induced by the Auger electrons emitted in the decay of the X-ray-induced holes produces mobile gas-phase charges that instantly neutralize any charge accumulating on the sample. This simple technique works very well over the whole range of X-ray energies (Schroeder et al., 1998) including the soft absorption edges (Adamczyk et al., 2000). Unfortunately the configuration of the beamline available in the current study precluded the use of this technique.

If the specimen is in powder form, the effect of charging on the XANES can be reduced by mixing the insulator with graphite (Nordberg et al., 1970) or carbon black (Obrovac et

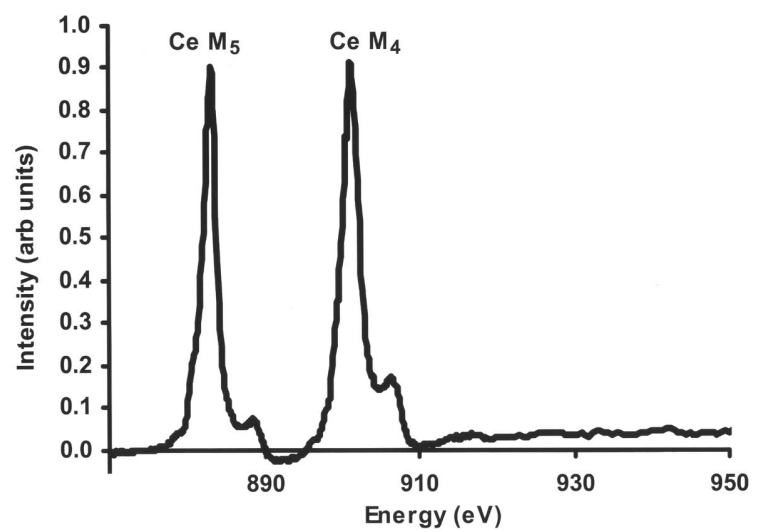

Figure 1

As-recorded Ce $M_{4,5}$-edge XANES from $40 \mathrm{wt} \%$ graphite and $60 \mathrm{wt} \%$ of $21 \mathrm{~mol} \% \mathrm{CeO}_{2}-\mathrm{ZrO}_{2}$ after background subtraction showing the nonphysical negative region between the white lines resulting from specimen charging. al., 1997), establishing an electrically conducting network through the sample. This technique was employed here. However, in some cases this does not solve the problem, as illustrated in Fig. 1. This figure shows the as-recorded Ce $M_{4,5^{-}}$ edge from a pellet composed of $35 \mathrm{wt} \%$ graphite and $65 \mathrm{wt} \%$ of $21 \mathrm{~mol} \% \mathrm{CeO}_{2}-\mathrm{ZrO}_{2}$. The background has been removed by subtracting the extrapolation of the signal prior to the edge. In the energy region between the $M_{5}$ and $M_{4}$ white lines, the signal intensity drops below the background level, i.e. the contribution from the edge apparently becomes negative. Clearly, this is an unphysical result and is a consequence of charging in the insulating grains of $21 \mathrm{~mol} \% \mathrm{CeO}_{2}-\mathrm{ZrO}_{2}$ despite the presence of conducting graphite powder.

The graphite introduces a second problem in that it gives rise to a strong carbon $K$-edge in the spectrum. As discussed in $\S 5$, the XANES and the extended X-ray absorption fine structure (EXAFS) from this edge both obscure subsequent weak edges and make background subtraction more difficult for the $\mathrm{O} K$-edge.

In this paper we address the correction of these effects in data obtained during a study of the XANES on the absorption edges in $\mathrm{ZrO}_{2}$ stabilized with a range of metal oxides $\left(\mathrm{Y}_{2} \mathrm{O}_{3}\right.$, $\mathrm{CeO}_{2}, \mathrm{HfO}_{2}$ and $\left.\mathrm{La}_{2} \mathrm{O}_{3}\right)$. All of the XANES measurements reported here were carried out using the TEY signal. Where possible, the XANES obtained was compared with the ELNES collected from the same specimens. In general, the relative energies of the peaks observed in the two techniques are in excellent agreement. However, the relative intensities of the features differ significantly. The discrepancy is greatest in regions where the TEY signal intensity changes most rapidly during the acquisition of the XANES data, i.e. immediately after the edge onset. This is the result of the time constants of the charging process, which are large in comparison with the dwell time at each energy. By measuring the TEY signal at equilibrium at an energy immediately below an ionization edge of interest and then suddenly stepping the energy onto the edge, the time dependence of the signal from the edge can be measured. This experimentally measured time dependence can then be fitted to an analytic function which is used to correct the XANES data.

\section{Experimental}

\subsection{Materials}

We have investigated a range of $\mathrm{ZrO}_{2}$ samples doped with 3, $5,8,10,15,20,25$ and $30 \mathrm{~mol} \% \mathrm{Y}_{2} \mathrm{O}_{3}, 10 \mathrm{~mol} \% \mathrm{La}_{2} \mathrm{O}_{3}, 10,21$, $50 \mathrm{~mol} \% \mathrm{CeO}_{2}$ and $10 \mathrm{~mol} \% \mathrm{HfO}_{2}$. Most of the samples were synthesized and supplied by MEL Chemicals. The 21 and $50 \mathrm{~mol} \% \mathrm{CeO}_{2}$ doped materials were supplied by Johnson Matthey. In addition, we investigated the corresponding pure oxides, i.e. $\mathrm{ZrO}_{2}, \mathrm{Y}_{2} \mathrm{O}_{3}, \mathrm{La}_{2} \mathrm{O}_{3}, \mathrm{CeO}_{2}$ and $\mathrm{HfO}_{2}$ (Alpha Aesar). All the samples were supplied in powder form. With the exception of the 3 and $5 \mathrm{~mol} \% \mathrm{Y}_{2} \mathrm{O}_{3}-\mathrm{ZrO}_{2}$ materials, $\mathrm{X}$-ray diffraction showed them to be single phase with lattice parameters consistent with their compositions. The 3 and $5 \mathrm{~mol} \% \mathrm{Y}_{2} \mathrm{O}_{3}-\mathrm{ZrO}_{2}$ powders were intended to be the stabi- 
lized tetragonal phase. Owing to the nature of the preparation process, small volume fractions of the cubic or monoclinic phase are often found in powders with these compositions. Here the $5 \mathrm{~mol} \%$ powder contained trace quantities of the stabilized cubic phase while the $3 \mathrm{~mol} \%$ sample contained $5-10 \%$ of monoclinic zirconia. At this level, the presence of the second phase has no effect on the results presented here.

For the XAS experiments, each sample was mixed and ground with graphite powder (Carbone of America, Ultra Carbon Division), then pressed into a disc using a hydraulic press, giving discs that were $1 \mathrm{~mm}$ thick and $13 \mathrm{~mm}$ in diameter. The discs of $\mathrm{Y}_{2} \mathrm{O}_{3}-\mathrm{ZrO}_{2}, \mathrm{CeO}_{2}-\mathrm{ZrO}_{2}, \mathrm{HfO}_{2}-\mathrm{ZrO}_{2}$ and $\mathrm{La}_{2} \mathrm{O}_{3}-\mathrm{ZrO}_{2}$ were formed using $40 \mathrm{wt} \%$ graphite. In order to ensure that the volume fraction was approximately constant, the amount of graphite mixed with the pure oxides varied: $35 \mathrm{wt} \%\left(\mathrm{CeO}_{2}\right), 25 \mathrm{wt} \%\left(\mathrm{HfO}_{2}\right), 40 \mathrm{wt} \%\left(\mathrm{La}_{2} \mathrm{O}_{3}\right)$ and $43 \mathrm{wt} \%\left(\mathrm{Y}_{2} \mathrm{O}_{3}\right)$. For the EELS experiments, the samples were ground in a mortar, dispersed in propanol and then agitated in an ultrasonic bath. Droplets of the suspension were then placed on a holey carbon-film-coated copper grid.

\subsection{XAS procedure}

The XAS experiments reported here were carried out at the Daresbury synchrotron radiation source using beamline 1.1. Beamline 1.1 is a soft X-ray beamline providing monochromatic photons over the energy range $200-1000 \mathrm{eV}$. In the experiments, a spherical-grating monochromator with 1800 lines $\mathrm{mm}^{-1}$ was used. Two-thirds of the grating was coated in gold and one-third in nickel. The nickel-coated section is used to reduce the second-order light content at energies above $\sim 425 \mathrm{eV}$, i.e. half of the $\mathrm{Ni} L_{3}$-edge threshold $(\sim 850 \mathrm{eV})$. For the oxygen $K$-edge experiments, data were recorded over the energy range $250-630 \mathrm{eV}$ using the Nicoated region of the monochromator. The energy range was chosen so that both the carbon and oxygen $K$-edges were present in the spectra obtained. The carbon $K$-edge is required for a number of reasons, as discussed below. On beamline 1.1 we also performed measurements at the $\mathrm{La}$ and $\mathrm{Ce} M_{4,5}$-edges over the ranges $790-950 \mathrm{eV}$ and $840-1000 \mathrm{eV}$, respectively. For these edges, the Au-coated area of the grating was used.

The TEY signal $\left(I_{\mathrm{e}}\right)$ was detected using an electrometer connected to the isolated sample holder, which was biased at $-56 \mathrm{~V}$ with respect to ground. In the presence of charging, this bias voltage plays an important role in keeping the signal level high. The incident-beam intensity $\left(I_{\mathrm{o}}\right)$ was monitored by measuring the drain current from a fine gold mesh biased at $-47 \mathrm{~V}$. This mesh was thoroughly ion-beam cleaned to remove surface contamination. There was no window (contamination barrier) between the gold mesh and the sample. The voltage outputs from the electrometers were converted to frequencies using voltage-to-frequency converters and the output pulse trains fed to counters that were enabled for the integration time chosen. All spectra were recorded with an integration time of $1 \mathrm{~s}$ per point. To minimize the time required to scan a spectrum, it was divided up into regions whose step size was
Table 1

Energy step used in each energy range when recording the $\mathrm{C}$ and $\mathrm{O} K$ edge XANES.

\begin{tabular}{lll}
\hline$E_{\text {initial }}(\mathrm{eV})$ & Step $(\mathrm{eV})$ & $E_{\text {final }}(\mathrm{eV})$ \\
\hline 250.00 & 1.00 & 280 \\
280.20 & 0.20 & 350 \\
352.00 & 2.00 & 430 \\
430.50 & 0.50 & 500 \\
500.10 & 0.10 & 570 \\
570.25 & 0.25 & 590 \\
590.50 & 0.50 & 620 \\
\hline
\end{tabular}

matched to the detail present in the signal. The set-up used for the carbon and oxygen $K$-edges is shown in Table 1 .

If the spectra obtained are to be corrected for the effect of specimen charging, it is important to be aware of the 'real' acquisition time per point. While the signal integration time was $1 \mathrm{~s}$, the average dwell time per point, i.e. the total acquisition time divided by the total number of points, was $2 \mathrm{~s}$. The difference between the integration and dwell times is due to the time required for the monochromator to settle at each new angular position. In addition, the charge state of the sample should ideally reach equilibrium before the start of the acquisition sequence to aid charge correction. To approach this, the monochromator was positioned at the starting energy and the specimen was irradiated at this energy for a period of at least $3 \mathrm{~min}$ before a scan was commenced.

Preliminary investigations also revealed that the XANES spectrum from an as-pressed pure graphite disc exhibited an oxygen $K$-edge of significant intensity. If the surface of the graphite disc was gently abraded by rubbing on filter paper, the oxygen signal was reduced to a low level. The spurious oxygen signal is clearly associated with the surface of graphite since it disappears when the surface layer is removed. However, there was no evidence of a rapid build-up of oxygen on the surface again if rubbed specimens spent time in air prior to insertion into the vacuum of the beamline. This suggests that the surface oxygen results from the disc-pressing process and is presumably there in all the specimen discs. To remove it, all discs were rubbed in this fashion prior to recording data. Fig. 2 compares the $\mathrm{O} K$-edge from a rubbed and an unrubbed disc made up from $70 \mathrm{wt} \%$ graphite and $30 \mathrm{wt} \%$ of $10 \mathrm{~mol} \% \mathrm{Y}_{2} \mathrm{O}_{3}-\mathrm{ZrO}_{2}$, showing that removal of this surface layer is crucial. In order that the residual contribution from the oxygen in the graphite can be neglected, it is important to have a high volume fraction of YSZ in the pellet. A pellet with $70 \mathrm{wt} \%$ graphite contains only $\sim 14 \mathrm{vol} \%$ of YSZ. Thus all subsequent work was performed with pellets containing $40 \mathrm{wt} \%$ graphite, which is equivalent to $37 \mathrm{vol} \%$ YSZ. Going to lower graphite fractions appeared to lead to a significant increase in the problems from charging.

\subsection{Measurement of specimen charging in XAS}

To monitor the extent of the charging effect and enable the time constants for the process to be determined, the response of the TEY signal to a sudden change in its magnitude is required. More specifically, the response of the signal from the 


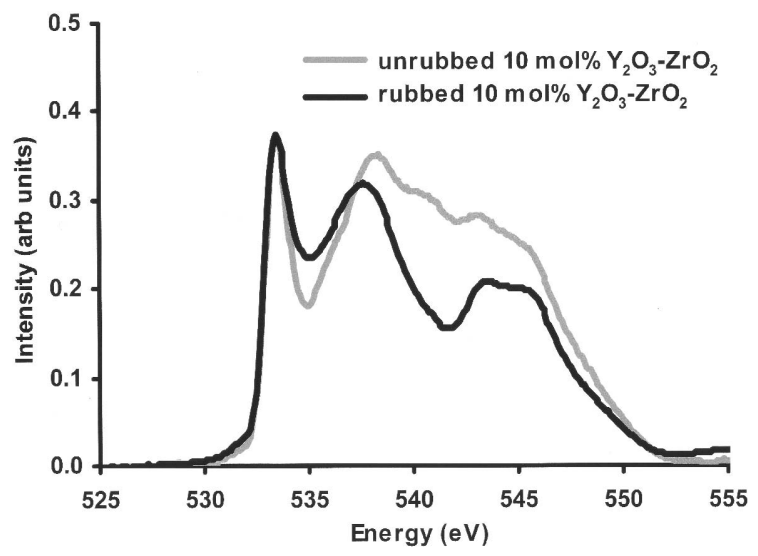

Figure 2

Comparison of the XANES O $K$-edge from a sample made up of $70 \mathrm{wt} \%$ graphite and $30 \mathrm{wt} \%$ of $10 \mathrm{~mol} \% \mathrm{Y}_{2} \mathrm{O}_{3}-\mathrm{ZrO}_{2}$, before and after removing the surface layer by rubbing on filter paper. The spectra are normalized to match at the first peak.

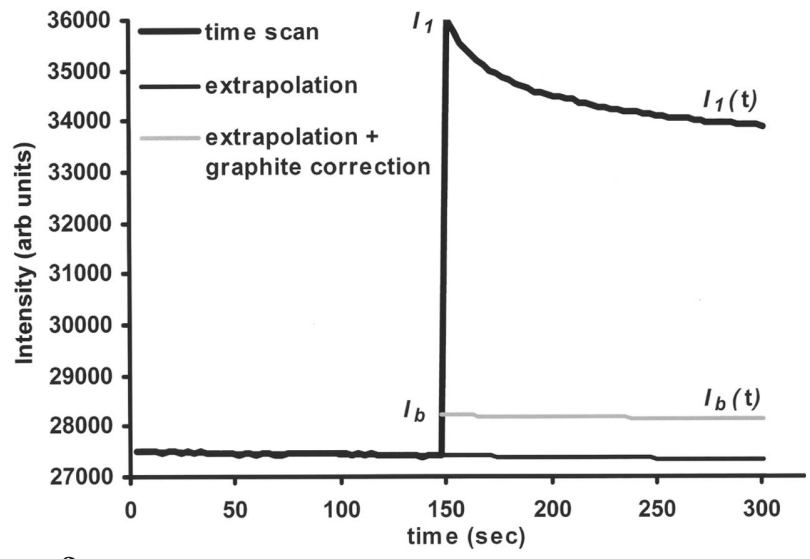

Figure 3

The time response of the total electron yield signal, $I_{\mathrm{e}}$, initially close to equilibrium at $531 \mathrm{eV}$ (the background prior to the $\mathrm{O} K$-edge) when the energy is changed to suddenly changed $533 \mathrm{eV}$ (the $\mathrm{O} K$-edge maximum) after $150 \mathrm{~s}\left(I_{1}\right)$. Also shown are the extrapolated background signal and the extrapolated background contribution corrected for the change in the graphite signal $\left(I_{\mathrm{b}}\right)$, as discussed in $\S 5$. The sample is made up of $40 \mathrm{wt} \%$ of graphite and $60 \mathrm{wt} \%$ of $3 \mathrm{~mol} \% \mathrm{Y}_{2} \mathrm{O}_{3}-\mathrm{ZrO}_{2}$.

material of interest must be identified. Thus the time response of an ionization edge is measured. This is achieved by measuring the TEY signal, $I_{\mathrm{e}}$, as a function of time using the time scan mode of acquisition. In this mode the incident energy is kept constant and the signal is repeatedly measured at fixed integration time. This time scan mode was modified so that, after a predetermined number of measurements at one incident energy, the energy was changed and a further sequence of intensities measured immediately after the change. By choosing an energy in the background region immediately before an absorption edge from the material of interest and one at the highest point on the edge, a large change in $I_{\mathrm{e}}$ is obtained. This time variation is dominated by that of the ionization edge since the change in the background contribution is very small over this energy step. For example, in the case of the oxygen $K$-edge, an initial energy of $\sim 531 \mathrm{eV}$ might be chosen, i.e. just prior to the onset of the absorption edge. $I_{\mathrm{e}}$ is measured for 50 integrations, each of $3 \mathrm{~s}$. The photon energy is then changed to a value of $\sim 533 \mathrm{eV}$ (i.e. on the maximum of the $\mathrm{O} K$-edge) in the shortest time the system will allow $(\sim 1 \mathrm{~s}) . I_{\mathrm{e}}$ is then measured for a further 50 integrations, each of $3 \mathrm{~s}$. Prior to starting the scan, the system is allowed to settle at the initial energy for a time longer than the time spent acquiring data at each energy. Fig. 3 shows one such time scan recorded from the $3 \mathrm{~mol} \% \mathrm{Y}_{2} \mathrm{O}_{3}-\mathrm{ZrO}_{2}$ sample. This is similar to the shape observed by Gilbert et al. (2000). At this point it should be noted that supplementary time scan measurements, using both the zirconia samples and a pure graphite sample, showed that there is no time dependence from the change of the signal from the graphite.

The accuracy of any correction procedure can only be validated by an independent check. As discussed above, the intensities in ELNES are not affected by any steady-state charging of the specimen. Hence the corrected XANES is compared with the ELNES from the same material.

\subsection{EELS procedure}

All the EELS measurements were carried out using a $100 \mathrm{kV}$ HB5 scanning transmission electron microscope (STEM) fitted with post-specimen lenses (Craven \& Buggy, 1981) and equipped with a GATAN 666 PEELS spectrometer and a Link Analytical energy-dispersive X-ray (EDX) spectrometer. The probe half-angle was $11 \mathrm{mrad}$, giving a probe diameter of $\sim 1 \mathrm{~nm}$ and a probe current of $\sim 0.2 \mathrm{nA}$. The collection semi-angle at the specimen was $12.5 \mathrm{mrad}$. During spectrum acquisition, the probe was scanned over an area $5.2 \mathrm{~nm} \times 3.8 \mathrm{~nm}$ to reduce the electron dose to the specimen. Details are given by Vlachos et al. (2001).

EDX spectroscopy was used to determine the dopant distribution in each sample. Preliminary results revealed that, while the composition within individual grains in a sample appeared to be homogeneous, there was significant compositional inhomogeneity between different grains in some of the powders (Vlachos et al., 2001). For this reason, an EDX spectrum was recorded from the same area as each set of EELS data to determine the local dopant concentration.

In the case of the $\mathrm{CeO}_{2}-\mathrm{ZrO}_{2}$ materials, significant radiation damage was observed. In this case, a sequence of spectra was acquired from an area that had only been imaged at relatively low magnification. Hence it had received an initial dose far lower than that required to record a spectrum. The beam blank system was used to stop the incident electrons reaching the specimen while the magnification was increased and the beam shift settings changed to previously determined values that placed the area of interest at the correct position. After the spectrometer had completed a detector readout, the beam was unblanked and the sequence of spectra recorded. By comparing the shapes of successive spectra in such a sequence, it was possible to identify the point at which a shape change was starting to occur and hence to make use of only those spectra acquired before that point was reached. 


\section{Modelling the charging process}

As described above, time scan measurements were performed to investigate the charging process. The purpose of these measurements was to measure the time response of the TEY signal, $I_{\mathrm{e}}$, at fixed energies, $E_{1}$ and $E_{2}$, prior to and on the absorption edge, respectively. The time scan in Fig. 3 shows that $I_{\mathrm{e}}$ varies slowly with time at the initial energy of $531 \mathrm{eV}$. Thus, even after a settling time of 3 min prior to starting the scan, the initial signal is still changing with time. Part of this is the steady decrease of the current in the synchrotron itself and part is the sample still coming to equilibrium as a result of charging processes with very long time constants. The direction of this residual settling depends on whether $I_{\mathrm{e}}$ at $531 \mathrm{eV}$ is greater or less than at the previous energy of irradiation. The rate of change depends on the time the sample has been at $531 \mathrm{eV}$ before the time scan was started and also on the sample, since the time constants vary from sample to sample. However, a long time constant has no effect on sharp features recorded over times that are short in comparison, and so these slow variations are neglected.

In order to correct the XANES measurements for charging, it is necessary to have a quantitative description of the timedependence of the TEY signal. To obtain a relatively simple correction procedure for a signal that is the result of a complex cascade process, a number of simplifying assumptions are made. Consideration of their limitations is discussed when the results are presented in later sections. As a first approximation, the sample is modelled as a capacitor. One electrode is connected to ground via an electrometer and a source of bias voltage, $V_{\mathrm{b}}$, and the other is in a vacuum and exposed to the incident photons. The latter electrode is taken to be sufficiently thin that it stops neither photons nor electrons. It is assumed that the dielectric gives a large but finite resistance between the plates. While highly idealized, this reproduces the situation in the powder sample reasonably well. At some points on a particle, the graphite is in contact with the surface while at others there is no direct contact with the graphite. However, any such isolated point on the surface of the particle will have a resistance (either surface or bulk) to the graphite. This resistance will allow any surface charge to dissipate eventually. Cazeau (1999) and Gilbert et al. (2000) have used the same basic approach to this problem.

The effect of the photon beam is to eject electrons from the exposed surface into the vacuum and equilibrium will occur when the ejected electrons are replaced by electrons flowing in from the graphite at the same rate. To create this current flow, the potential of the surface must increase relative to the graphite. This, in turn, will mean that only electrons with kinetic energy in excess of the potential barrier created will escape into the vacuum and so the number escaping will decrease as the surface charge increases. At some surface potential distribution, equilibrium is reached.

The inset in Fig. 4 shows the equivalent circuit where a photon flux causes ejection of electrons from the surface at a rate corresponding to a conventional current, $I_{\mathrm{e}}$, flowing onto the top plate of the capacitor. This results in a current, $I_{\mathrm{e}}$, flowing through the electrometer to ground. $I_{\mathrm{e}}$ is made up of two components. $I_{\mathrm{c}}$ increases the charge, $q$, on the capacitor while $I_{\mathrm{R}}$ flows through the resistance between the plates. A bias voltage, $V_{\mathrm{b}}$, is applied to the lower plate. The voltage of the top surface with respect to ground is $V_{\mathrm{s}}$. As the capacitor gains charge, $q$, the potential difference across the plates, $V_{\mathrm{c}}=$ $\left(V_{\mathrm{s}}-V_{\mathrm{b}}\right)$, rises and the current through the resistance, $R$, between the plates increases. At equilibrium, the voltage of the top surface is $V_{\mathrm{so}}, I_{\mathrm{c}}$ is zero and $I_{\mathrm{R}}$ is equal to $I_{\mathrm{e}}$.

Fig. 4 shows schematically the variation of $I_{\mathrm{e}}$ with $V_{\mathrm{s}}$ at constant incident photon energy and intensity. The secondary electrons emitted from the surface will give the curve a shape similar to that from a thermionic diode or vacuum photodiode, i.e. $I_{\mathrm{e}}$ rises to saturation as $V_{\mathrm{s}}$ becomes negative and falls as $V_{\mathrm{s}}$ becomes positive. To achieve cut-off, $V_{\mathrm{s}}$ would have to rise to a value consistent with the energy of the most energetic electron created in the absorption process.

One approach to finding a simple functional form for the process is to assume that the curve in Fig. 4 can be approximated as a straight line over the range of parameters encountered. Thus $I_{\mathrm{e}}=I_{\mathrm{eo}}-V_{\mathrm{s}} / r$ where $r$ is a dynamic resistance, given by the inverse of $\partial I_{\mathrm{e}} / \partial V_{\mathrm{s}}$ evaluated at the equilibrium value of $V_{\text {so. }}$.

The magnitude of $I_{\mathrm{e}}$ depends on the incident flux and also on the incident photon energy via the absorption coefficient in the material. If either the incident flux or the absorption coefficient increases, the curve increases in height and possibly changes in shape. If the material is insulating so that $R$ is large, an increase in flux or absorption will result in an increase in the charge on the capacitor. This increases $V_{\mathrm{c}}$ and hence $V_{\mathrm{s}}$, resulting in a drop in $I_{\mathrm{e}}$ until equilibrium is re-established at a new value of $V_{\text {so. }}$.

If it is assumed that $r$ remains constant, it is possible to show that a sudden change in photon energy across an absorption edge, as in Fig. 3, will result in an initial step increase in the TEY signal followed by an exponential decay to the equilibrium value so that

$$
I_{\mathrm{e}}(t) / I_{\mathrm{eo}}=(1-g)+g \exp (-\alpha t)
$$

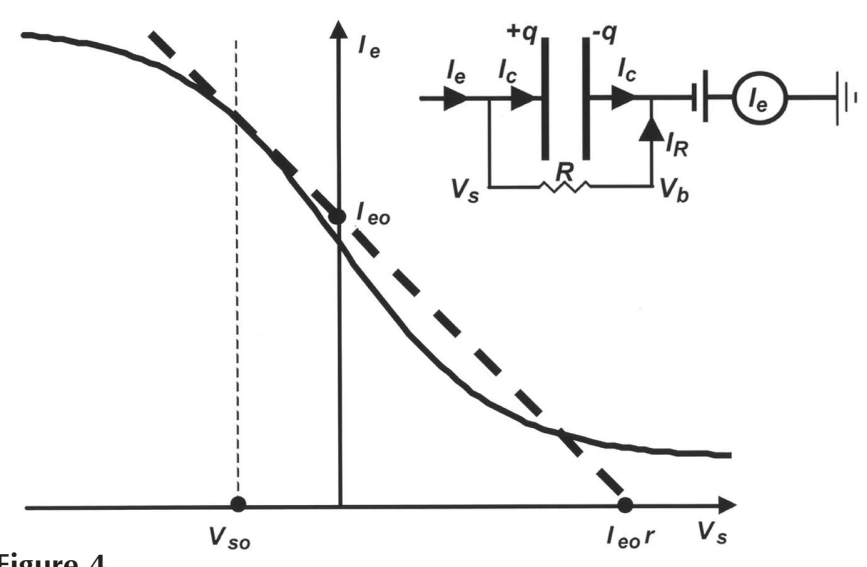

Figure 4

A schematic diagram showing the variation of $I_{\mathrm{e}}$ with the surface potential $V_{\mathrm{s}}$. The equivalent circuit for the charging process is shown in the inset. 
where $I_{\mathrm{eo}}$ is the initial change of the drain current to ground and $I_{\mathrm{e}}(t)$ is the change a time, $t$, later. $\alpha$ is an inverse time constant and $g$ is a measure of the magnitude of the charging.

However, $r$ will vary with $V_{\mathrm{s}}$. Moreover, $R$ and the capacitance will vary from particle to particle depending on the size and the contact made by the surrounding graphite. Cazeau (1999) notes this problem and Gilbert et al. (2000) develop an implicit expression for the current density as a function of time for an idealized system. However, their expression cannot be used as a simple fitting function to describe the experimental time dependence. It is clear that equation (1) is unlikely to describe the experimental observations sufficiently well. However, since only an accurate parameterization of the time variation is required below, equation (1) was simply extended to allow for more than one time constant giving

$$
I_{\mathrm{e}}(t) / I_{\mathrm{eo}}=1-\sum_{i} g_{i}+\sum_{i} g_{i} \exp \left(-\alpha_{i} \Delta t\right)
$$

Using two time constants, equation (2) resulted in an excellent agreement with the experimental data, as shown in Fig. 5. Here the experimental points are derived from the data in Fig. 3 by forming the reduced quantity $\left[I_{1}(t)-I_{\mathrm{b}}(t)\right] /\left(I_{1}-I_{\mathrm{b}}\right)$ where $I_{\mathrm{b}}(t)$ is the extrapolated background signal corrected for any change in the graphite signal, as discussed in $§ 5$. The deviation between the experimental data and the fit is less than $0.4 \%$, as shown in the inset.

Typical values for the two time constants of the $\mathrm{O} K$-edge are $15 \mathrm{~s}$ and $100 \mathrm{~s}$ but the values obtained for a given sample can vary by up to $25 \%$ depending on the area of the sample irradiated. There is also some dependence of the time constants on the $\mathrm{Y}_{2} \mathrm{O}_{3}$ fraction, with the end members of the series $\left(0 \%\right.$ and $\left.30 \% \mathrm{Y}_{2} \mathrm{O}_{3}\right)$ having the shortest time constants while the mid-range compositions have the longest. Thus the charging behaviour is clearly a local property of the particular specimen as well as a property of the material itself.

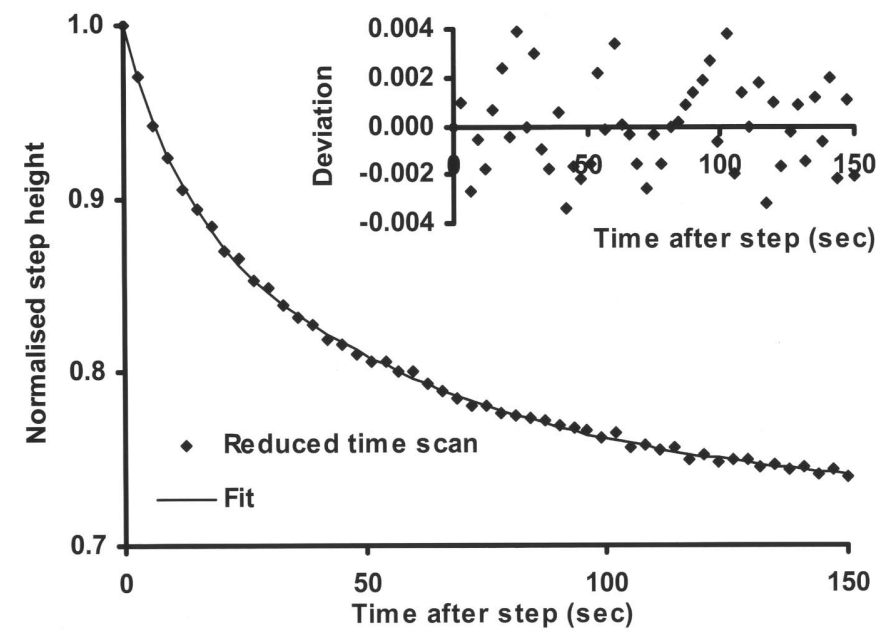

Figure 5

Fit of equation (2) with two sets of charging constants to the reduced form of the data in Fig. 3 obtained by forming the quantity $\left[I_{1}(t)-I_{\mathrm{b}}(t)\right] /\left(I_{1}-I_{\mathrm{b}}\right)$ where $I_{\mathrm{b}}(t)$ is the extrapolated background signal after correction for the graphite signal. The deviation between the experimental data and the fit is shown in the inset.

\section{An algorithm for correcting for the effect of charging}

Having established a simple expression for the time dependence of the charging process, a scheme to correct for the effects of charging can be developed. In the absence of charging, the signal is independent of time unless the energy of the incident radiation changes. Thus if the scan process is approximated to a series of steps in energy, the signal observed would be of the form of $I_{\mathrm{u}}(t)$, as shown schematically in Fig. 6 for the case where the signal increases with energy. If the specimen charges, then the signal will reach the level appropriate to the equilibrium charge state of the specimen if the energy is kept constant for a period long compared with the longest time constant of the charging process. This equilibrium signal would have the form of $I_{\infty}(t)$ in Fig. 6. The time constants of $15 \mathrm{~s}$ and $100 \mathrm{~s}$ are long compared with the time spent at each energy in the scan $(\sim 2 \mathrm{~s})$. Indeed to allow the signal to reach equilibrium at each point would require an increase in the time per scan from $\sim 1 \mathrm{~h}$ to $>>50 \mathrm{~h}$.

Equation (2) shows that the charging process will result in $I(t)$ approaching the equilibrium value, $I_{\infty}(t)$, in a way that can be described by a sum of exponentially decaying terms. However, for the purpose of deriving the form of the correction necessary, it is assumed that the effect of the charging process can be represented by a single exponential term. The additional time constants will be included at the end as a simple extension to the procedure.

If the system starts in equilibrium at energy $E_{0}$, and the energy suddenly changes to $E_{1}$, then there will be an initial change in the signal, $\Delta I_{\mathrm{u}}^{1}$. This will be the same change that would have occurred in the absence of charging since the sample has not had time to change its charge state. As the sample charges, the signal will change with time according to (1) giving

$$
\Delta I^{1}(t)=\Delta I_{\mathrm{u}}^{1}[(1-g)+g \exp (-\alpha t)],
$$

which will eventually decay to $\Delta I_{\infty}^{1}=\Delta I_{\mathrm{u}}^{1}(1-g)$. Thus, at time $t$, the signal is $\left[\Delta I_{\mathrm{u}}^{1} g \exp (-\alpha t)\right]$ from the equilibrium value.

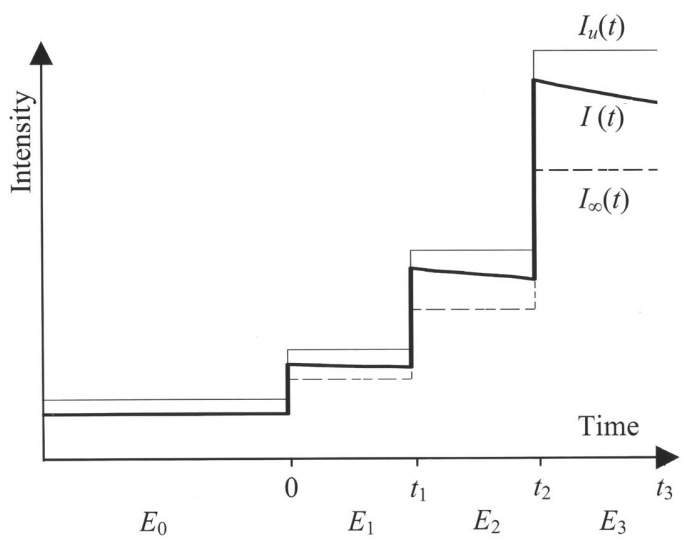

Figure 6

Schematic diagram showing the signal obtained as the energy is incremented for three conditions: in the absence of charging, $I_{\mathrm{u}}(t)$ (fine line); with a charging sample coming to equilibrium at each energy step, $I_{\infty}(t)$ (dashed line); when the sample charges over a period that is long compared with the time at each energy, $I(t)$ (thick line). 
If after a time interval $\Delta t$ the energy is changed from $E_{1}$ to $E_{2}$, the signal will increase by a further $\Delta I_{\mathrm{u}}^{2}$. Again this is the change that would have occurred in the absence of charging. However, the new equilibrium value will be $\left(\Delta I_{\mathrm{u}}^{2}+\Delta I_{\mathrm{u}}^{1}\right)(1-g)$ above the value at $E_{0}$. Hence the signal will differ from the new equilibrium value by $\left[\Delta I_{\mathrm{u}}^{2} g+\Delta I_{\mathrm{u}}^{1} g \exp (-\alpha \Delta t)\right]$. The difference from the new equilibrium value will continue to decay exponentially so that, at time $t$ after the energy first changed from $E_{0}$ to $E_{1}$, it will be $\left\{\Delta I_{\mathrm{u}}^{2} g \exp [-\alpha(t-\Delta t)]+\Delta I_{\mathrm{u}}^{1} g \exp (-\alpha t)\right\}$. Each additional energy step will add a further deviation from equilibrium. Thus the overall deviation of the signal from the current equilibrium value will be a sum of terms. Each term will be the product of the initial signal change caused by the energy step, $\Delta I_{\mathrm{u}}^{i}$, multiplied by an amplitude term, $g$, and further multiplied by an exponential factor whose exponent is determined by the time elapsed since the energy step occurred. Hence for a series of energy steps at time intervals of $\Delta t$,

$$
\begin{aligned}
I(N \Delta t) & =I_{\infty}(N \Delta t)+\sum_{n=1}^{N} \Delta I_{\mathrm{u}}^{n} g \exp [-\alpha(N-n) \Delta t] \\
& =I_{\mathrm{u}}(N \Delta t)(1-g)+\sum_{n=1}^{N} \Delta I_{\mathrm{u}}^{n} g \exp [-\alpha(N-n) \Delta t] .
\end{aligned}
$$

For the charging process, $\alpha \Delta t$ is much less than unity and so the sum can be replaced by an integral, allowing easier manipulation. For this, $n \Delta t$ is replaced by $t^{\prime}$, the time at which the energy change occurred, and $\Delta I_{\mathrm{u}}^{n}$ is replaced by $\left(\mathrm{d} I_{\mathrm{u}} / \mathrm{d} t^{\prime}\right) \mathrm{d} t^{\prime}$ where the derivative is evaluated at $t^{\prime}$ giving

$$
I(t)=I_{\mathrm{u}}(t)(1-g)+g \int_{0}^{t} \frac{\mathrm{d} I_{\mathrm{u}}}{\mathrm{d} t^{\prime}} \exp \left[-\alpha\left(t-t^{\prime}\right)\right] \mathrm{d} t^{\prime} .
$$

This can be integrated by parts and re-arranged to give

$$
I_{\mathrm{u}}(t)=I(t)+g I_{\mathrm{u}}(0) \exp (-\alpha t)+g \alpha \int_{0}^{t} I_{\mathrm{u}}\left(t^{\prime}\right) \exp \left[-\alpha\left(t-t^{\prime}\right)\right] \mathrm{d} t^{\prime}
$$

Thus the signal, $I_{\mathrm{u}}(t)$, that would have been obtained in the absence of charging can be obtained from the measured signal, $I(t)$, by the addition of two correction terms since the parameters $g$ and $\alpha$, which describe the effects of the charging, can be found from the time scan.

The first correction term can be evaluated directly at time $t$, since $I_{\mathrm{u}}(0)$ is just the equilibrium signal before the start of the scan, $I_{\infty}(0)$, divided by $(1-g)$. However, the second term involves the values of $I_{\mathrm{u}}\left(t^{\prime}\right)$ up to the current time, $t$. By starting with $I_{\mathrm{u}}(0)$, the value of $I_{\mathrm{u}}\left(t_{1}\right)$ can be evaluated. Using this, the value of $I_{\mathrm{u}}\left(t_{2}\right)$ can be evaluated and so on. Since the data obtained are discrete, equation (6) is replaced by a sum so that

$$
\begin{aligned}
I_{\mathrm{u}}(N \Delta t)= & I(N \Delta t)+g I_{\mathrm{u}}(0) \exp (-\alpha N \Delta t) \\
& +g \alpha \Delta t \sum_{n=1}^{N-1} I_{\mathrm{u}}(n \Delta t) \exp [-(N-n) \alpha \Delta t] .
\end{aligned}
$$

Here the upper limit of the sum is $(N-1)$ since it only involves values prior to the current time.
If the time response of the signal involves more than one time constant, equation (3) can be generalized to

$$
\Delta I^{1}(t)=\Delta I_{\mathrm{u}}^{1}\left[\left(1-\sum_{i} g_{i}\right)+\sum_{i} g_{i} \exp \left(-\alpha_{i} t\right)\right]
$$

The linear form of the equations then allows (7) to be rewritten as

$$
\begin{aligned}
I_{\mathrm{u}}(N \Delta t)= & I(N \Delta t)+I_{\mathrm{u}}(0) \sum_{i} g_{i} \exp \left(-\alpha_{i} N \Delta t\right) \\
& +\sum_{i} g_{i} \alpha_{i} \Delta t \sum_{n=1}^{N-1} I_{\mathrm{u}}(n \Delta t) \exp \left[-(N-n) \alpha_{i} \Delta t\right] .
\end{aligned}
$$

In principle, the algorithm should be applied to data that have an equilibrium value of the signal in the first channel to give the true corrected signal. However, if the algorithm is applied starting at an arbitrary point in the scan, the corrected signal approaches the true corrected signal after a number of data points which corresponds to an acquisition time of several times the longest time constant involved.

\section{Dealing with the background}

In the experimental data, $I_{\mathrm{e}} / I_{\mathrm{o}}$, the edge of interest sits on a background due to excitations of less tightly bound electrons in both the material of interest and the graphite. If the edge of interest is sufficiently far from other edges, the background preceding it is smooth and relatively slowly varying. By fitting a power-law function in the region before the edge, the function can be extrapolated under the edge and subtracted.

Provided there is no element on the surface of the optics having an edge in this vicinity, the change of $I_{\mathrm{o}}$ with photon energy, and hence with time, will be slow. Thus the part of $I_{\mathrm{e}}$ giving rise to the background will also change slowly with time. Hence, charging will have little or no effect on the background as equation (5) shows that the effect of charging is controlled by $\mathrm{d} I_{\mathrm{e}} / \mathrm{d} E$. Thus, under these circumstances the background can be removed either before or after correcting for the effects of charging. These conditions are satisfied for all edges of interest here apart from the $\mathrm{O} K$-edge.

For the $\mathrm{O} K$-edge, neither condition is satisfied since the EXAFS from the $\mathrm{C} K$-edge perturbs the background shape while the oxygen on the beam optics causes a significant variation of $I_{\mathrm{o}}$ in the vicinity of the edge. The effect of the EXAFS can be removed by recording the $\mathrm{C} K$-edge from a pure graphite specimen, scaling it to match the $\mathrm{C} K$-edge from the zirconia-containing specimen and subtracting. In principle, this gives the $\mathrm{O} K$-edge signal from the zirconia alone. The effects of charging must be corrected at this point and the background from the zirconia removed subsequently. The following procedure was used for the background subtraction. The spectrum of the pure carbon sample was scaled and subtracted from that of the zirconia sample. The shape of the $\mathrm{Zr}$ and $\mathrm{Y} M_{2,3}$-edges near to $330 \mathrm{eV}$ and $300 \mathrm{eV}$, respectively, allow the correct scaling factor to be determined. It is then possible to fit a reasonable power-law background in the region prior to the $\mathrm{O} K$-edge and extrapolate it under the edge 
to remove the background. The result still depends on the fitting window used but to a far lesser extent than if the graphite contribution had not been removed.

Returning to the time scan of Fig. 3, the initial change in $I_{\mathrm{e}}$ caused by the change in energy is composed of two parts, one from the graphite and one from the zirconia. The part from the graphite has no time dependence and, to find the change owing to the contribution from the zirconia alone, a correction must be made to the background signal. The values of $I_{\mathrm{e}} / I_{\mathrm{o}}$ for the graphite at the two energies in the time scan are known from the scaled graphite spectrum discussed in the previous paragraph and these values are very similar. However, the value of $I_{\mathrm{e}}$ itself changes significantly because of the change of $I_{\mathrm{o}}$ resulting from oxygen on the optics. Thus the change in $I_{\mathrm{e}}$ from the graphite can be obtained simply by multiplying the value of $I_{\mathrm{e}} / I_{o}$ from the graphite by the change of $I_{\mathrm{o}}$ over the energy step in the time scan. This change in $I_{\mathrm{e}}$ from the graphite is then used to correct the base line of the time scan, as shown in Fig. 3.

\section{Correction of the effects of charging}

\subsection{Ce and La $\mathbf{M}_{4,5}$-edges}

In the case of $\mathrm{La}$ and $\mathrm{Ce} M_{4,5}$-edges, the time scans were performed on the $M_{5}$-edges. The time scans were fitted using two time constants. Spectra from a pure graphite sample showed that the background was indeed smooth across the edges. The data were charge corrected and the background removed subsequently. Fig. 7 compares the $\mathrm{Ce} M_{4,5}$-edges from the $21 \mathrm{~mol} \% \mathrm{CeO}_{2}-\mathrm{ZrO}_{2}$ sample recorded by XAS before and after charge correction. (The uncorrected data were shown in Fig. 1.) The spectra are normalized in the region from 80 to $110 \mathrm{eV}$ after the $M_{5}$ white line. In the corrected spectrum, the white-line intensity is reduced and the unphysical negative region between the white lines is removed.

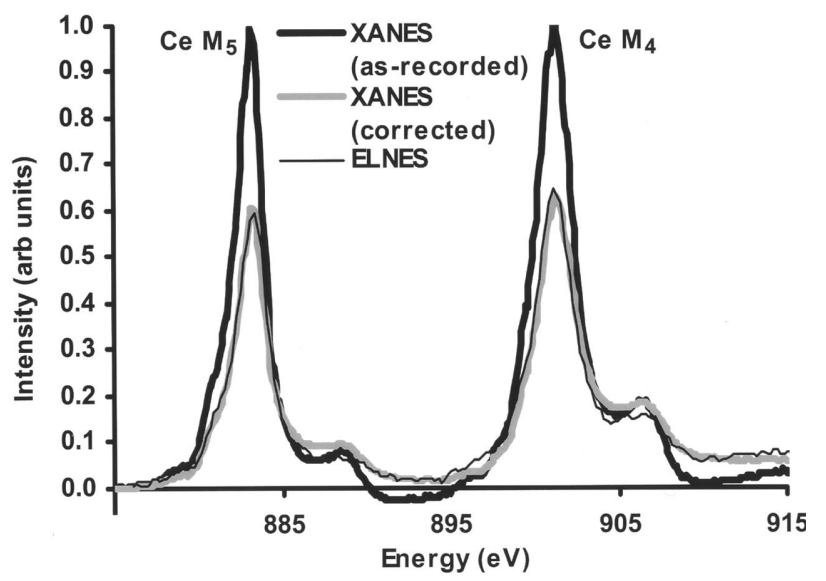

Figure 7

The Ce $M_{4,5}$-edge XANES from $40 \mathrm{wt} \%$ graphite and $60 \mathrm{wt} \%$ of $21 \mathrm{~mol} \% \mathrm{CeO}_{2}-\mathrm{ZrO}_{2}$ before and after correction for specimen charging compared with the ELNES from the Ce $M_{4,5}$-edge from $21 \mathrm{~mol} \% \mathrm{CeO}_{2}-$ $\mathrm{ZrO}_{2}$. All have had the background subtracted.
Also shown are the edges recorded by EELS normalized to the Ce $M_{5}$ peak intensity. It is quite clear that the agreement between the charge-corrected XANES data and the ELNES data is excellent, showing that the charge-correction algorithm works well for this edge. There are two areas of minor discrepancy. The first is that the continuum in the EELS data is high in the range $910-940 \mathrm{eV}$. This may be related to residual multiple scattering left by the deconvolution procedure used in processing the EELS data. The procedure assumes that the data are taken from an area of uniform thickness. In practice, the electron beam is scanned over a small area of the specimen during acquisition. Since the doped zirconia is in the form of small particles, this means that signals from a range of thicknesses may contribute to the spectrum and could perturb the deconvolution slightly. There is a second minor discrepancy. The intensity of the high-energy shoulders of the white lines in the fully corrected XANES is higher than in the ELNES while the reverse is true for the low-energy shoulders. This material undergoes radiation damage in the electron beam and one effect of this is to reduce the intensity of the high-energy shoulder and increase that of the low-energy shoulder. Thus the EELS data are probably showing the early stages of radiation damage.

\subsection{The $\mathrm{O} K$-edge}

For the $\mathrm{O} K$-edges, two time constants were again used to fit the times scans. Once the graphite contribution to the spectrum has been removed, as described in $\S 5$, the algorithm for correcting the effects of charging is applied. Fig. 8 compares the XANES $\mathrm{O} K$-edge shape from the $25 \% \quad \mathrm{Y}_{2} \mathrm{O}_{3}-\mathrm{ZrO}_{2}$ sample after background subtraction for the following cases:

(a) without correction for the graphite contribution or charging (GU-CU);

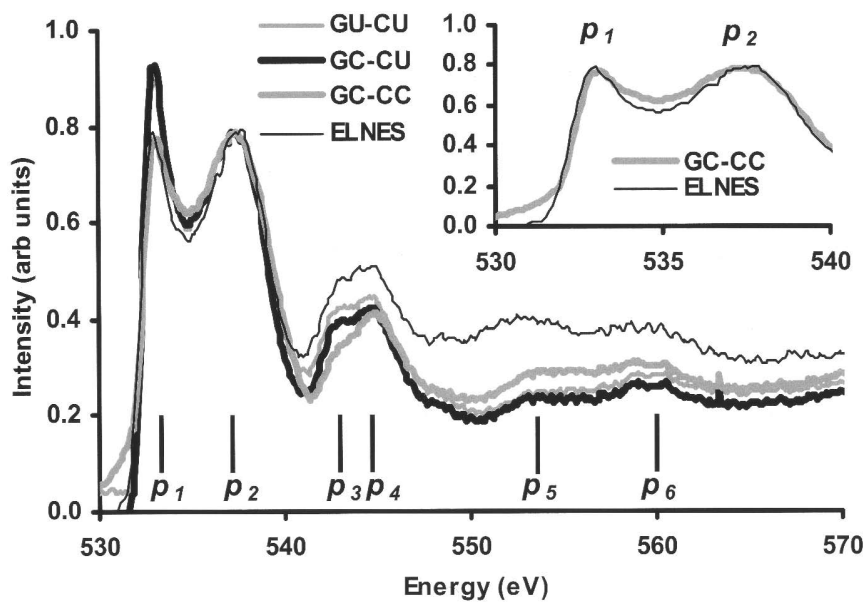

Figure 8

Comparison of the O $K$-edge XANES from $40 \mathrm{wt} \%$ graphite and $60 \mathrm{wt} \%$ of $25 \mathrm{~mol} \% \mathrm{Y}_{2} \mathrm{O}_{3}-\mathrm{ZrO}_{2}$ at each stage of processing. GU-CU: graphite uncorrected, charged uncorrected; GC-CU: graphite corrected, charged uncorrected; GC-CC: graphite corrected, charged corrected. Also shown is the ELNES from $25 \mathrm{~mol} \% \mathrm{Y}_{2} \mathrm{O}_{3}-\mathrm{ZrO}_{2}$. All have had the background subtracted. 
(b) after correction for the graphite contribution but without correction for charging (GC-CU);

(c) after correction for both the graphite contribution and charging (GC-CC).

The $\mathrm{O}-K \mathrm{XANES}$ is characterized by a number of peaks, as shown in Fig. 8. The edges have been normalized at the second peak, $p_{2}$, at about $538 \mathrm{eV}$. The effect of the graphite subtraction has little effect on the shape here whereas the charge correction significantly lowers the intensity of the first peak $p_{1}$ and also that of peak $p_{3}$.

The ELNES shape is also plotted in Fig. 8, again normalized to $p_{2}$. There is excellent agreement with the intensities of $p_{1}$ and $p_{2}$ in the charge-corrected XANES spectrum and the ELNES spectrum. In addition, the shapes of $p_{3}$ and $p_{4}$ are in better agreement than with the data uncorrected for charging. There is again a significant difference in the overall intensity between 540 and $570 \mathrm{eV}$, after which there is reasonable agreement given the uncertainties associated with background extrapolation. As well as the possibility of a residual error from the deconvolution process in the EELS data, there is a further potential source of difference in this energy region. The grid used to monitor $I_{\mathrm{o}}$ is made of gold, which has an $\mathrm{N}_{5^{-}}$ edge at $545 \mathrm{eV}$. This will cause a small increase in $I_{\mathrm{o}}$ with no corresponding increase in $I_{\mathrm{e}}$ and hence a reduction in $I_{\mathrm{e}} / I_{\mathrm{o}}$. The effect of this reduction will be magnified by the background-subtraction process and partially explains the lower XANES signal in Fig. 8.

The agreement between the $p_{1} / p_{2}$ intensity ratio in the ELNES and that in the XANES always improved after correction for charging. In general, the XANES for a given material showed a systematic increase in this ratio the later the data were taken in the experimental run, so that the agreement with the ELNES became worse. The later the data were taken, the bigger the change. The factor which correlates most strongly with this observation is the time since the cleaning of the gold mesh used to monitor $I_{\mathrm{o}}$. There is a significant change with time of the shape of the $I_{\mathrm{o}}$ versus $E$ curve in the vicinity of the $\mathrm{O} K$-edge and the effect may be the result of a small error in the normalized ratio $I_{\mathrm{e}} / I_{\mathrm{o}}$ intensity resulting from this. Confirmation would require a more detailed investigation of the effect.

\section{Discussion}

It is clear that there are a large number of factors that can perturb the XANES from these highly insulating materials. Fig. 2 showed that surface oxygen can play a major role if not removed. While all samples were treated in the same way, there is no guarantee that all had this spurious oxygen removed to the same degree. However, the samples were not examined in order of composition but followed the general trend of $0,5,10,20,30,3,8,15,25 \mathrm{~mol}_{\%} \mathrm{Y}_{2} \mathrm{O}_{3}$ to ensure that the whole range of compositions was covered in the event of a problem with the SRS limiting the time available. In addition to these compositions, the specimens containing the other dopants were also examined. At least two spectra were recorded from each sample at different times in the experi- mental session. The scatter at each composition is related to the systematic growth of contamination on the gold grid used to measure $I_{\mathrm{o}}$. Data taken later in the session show bigger discrepancies from the equivalent ELNES.

Given the particle-to-particle variation in the $\mathrm{Y} /(\mathrm{Y}+\mathrm{Zr})$ ratio in the 20 and $25 \mathrm{~mol} \% \mathrm{Y}_{2} \mathrm{O}_{3}-\mathrm{ZrO}_{2}$ samples, it would be expected that $p_{2}$ in the XANES from these materials, which averages over many particles, would be wider than that from the ELNES, for which only data with $\mathrm{Y} /(\mathrm{Y}+\mathrm{Zr})$ close to the nominal values are included. There is some indication of this in the inset of Fig. 8 where the minimum between $p_{1}$ and $p_{2}$ is shallower in the XANES than in the ELNES. This could be the result of the broadening of $p_{2}$ but confirmation of this would need additional work.

While the charge-correction algorithm works well near the threshold from which the time scan was recorded, there is evidence that it works less well away from the threshold. Some small background artefacts were observed when the scan rate changed or when the second-order radiation was suddenly removed at $430 \mathrm{eV}$ by the $\mathrm{Ni}$ coating on the grating. These artefacts occur because the processes generating the TEY signal from the background differ significantly from those generating the TEY signal from the $\mathrm{O} K$-edge. At the threshold of the $\mathrm{O} K$-edge, the main contribution to the TEY signal from $\mathrm{O}$ is from the $\mathrm{O}$ Auger electrons, with the photoelectrons having little energy to create secondary electrons. On the other hand, the main contribution to the TEY signal from the background prior to the $\mathrm{O} K$-edge is from excitation of the $\mathrm{Zr} M_{4,5}$-edges since the contribution from the graphite has already been removed. Here the first-order radiation creates photoelectrons and Auger electrons of similar energy. When second-order radiation is present below $430 \mathrm{eV}$ it will create additional photoelectrons of much higher energy than the Auger electrons. Thus it is not surprising that $\alpha$ and $g$ determined specifically for the $\mathrm{O} K$-edge near its threshold do not describe the behaviour of the background exactly, leading to the small artefacts observed.

Finally, Gilbert et al. (2000) have investigated the illumination of the specimen with light whose photon energy is bigger than the band gap of the material but less than the work function. In principle, this introduces photoconductivity without additional signal and so reduces the charging. They showed an effect on their highly insulating biological specimens but not sufficient to stop the charging. However, it is certainly worth following up for material like zirconia.

\section{Conclusions}

XANES from an insulating powder recorded by the TEY method can show a number of artefacts, particularly those related to charging. To obtain reasonable data it is essential to bias the sample negative with respect to ground. It is also essential to mix the insulating material with a finely divided conductor whose ionization edges will not interfere with the edges to be measured from the material of interest. However, the volume fraction of material should be high enough to maximize the signal from the edges of interest but not so high 
as to exacerbate the effects of charging by causing flashovertype effects on its surface. It is crucial that any surface contaminants that interfere with the edge of interest are removed, oxygen being of most concern here. It was found that the hydraulic press used to make the pellets introduces a surface layer of high oxygen content and this has to be removed.

The contribution from the graphite used to minimize the charging can be removed by scaled subtraction of a spectrum from pure graphite. The time dependence of the charging process can be determined from a time scan in which the energy is switched from just below the edge of interest to the maximum intensity on the edge at a point in the middle of the scan. This time dependence can be parameterized and used in an algorithm that corrects for the effects of the charging. This procedure is satisfactory close to the edge threshold but is only approximate elsewhere.

Even after all the corrections were made, there were still systematic differences between the ELNES and the XANES. There was also a deviation in overall intensity rather than shape in the region $10-50 \mathrm{eV}$ above the edge. One contribution to this is the difficulty of correcting for the multiple scattering in the ELNES when the sample thickness is nonuniform. Other contributions may come from residual artefacts in the XANES from the change in the energy of the photoelectron as a function of the energy in the spectrum or from the effect of an absorption edge in the monitor grid or from the effect of contamination in the monitor grid. Another important difference between XANES and ELNES is that the first technique averages the intensity over a large area of the sample while the latter can be used to record spectra from individual particles owing to its inherent high spatial resolution.

As methods of calculation improve, it is essential that such artefacts in the experimental data are removed so that it is clear where the remaining differences lie. It is also important that inter-comparison of different XANES measurements, or comparisons of XANES and ELNES, from insulating materials take into account the effects of charging if the XANES are recorded using the TEY method

Overall, given the number of effects involved in the XANES method, ELNES must be considered a simpler and more reliable technique for these relatively low-energy edges provided that suitable thin specimens can be made and that radiation damage is not a limiting factor. However, where signal-to-noise ratio, energy resolution and radiation damage are the key factors, XANES offers significant benefits provided the artefacts can be corrected.

The authors would like to thank Ian Kirkman and Sunil Patel (Daresbury), Jim Gallagher and Sam McFadzean for their help in this work. We are grateful to EPSRC (GR/ L66953), MEL Chemicals and Johnson Matthey for their support in carrying out this work

\section{References}

Adamczyk, B., Boese, O., Weiher, N., Schroeder, S. \& Kemnitz, E. (2000). J. Fluor. Chem. 101, 239-246.

Cazeau, J. (1999). J. Electron Spectrosc. Relat. Phenom. 105, 155-185.

Craven, A. J. \& Buggy, T. W. (1981). Ultramicroscopy, 7, 27-37.

Cros, A. (1992). J. Electron Spectrosc. Relat. Phenom. 59, 1-14.

Disko, M. M. (1992). Transmission Electron Energy Loss Spectrometry in Materials Science, edited by M. M. Disko, C. C. Ahn and B. Fultz. Warrendale: TMS.

Egerton, R. F. (1986). Electron Energy Loss Spectroscopy in the Electron Microscope. New York/London: Plenum.

Elam, W. T., Kirkland, J. P., Neiser, R. A. \& Wolf, P. D. (1988). Phys. Rev. $B, \mathbf{3 8}, 26-30$.

Erbil, A., Cargill, G. S. III, Frahm, R. \& Boehme, R. F. (1988). Phys. Rev. B, 37, 2450-2464.

Gilbert, B., Andres, R., Perfetti, P., Margaritondo G., Rempfer, G. \& de Stasio, G. (2000). Ultramicroscopy, 83, 129-139.

Groot, F. M. F. de (1994). J. Electron Spectrosc. Relat. Phenom. 67, 529-622.

Henke, B. L., Knauer, J. P. \& Premaratne, K. (1981). J. Appl. Phys. 52, 1509-1520.

Henke, B. L., Liesegang, J. \& Smith, S. D. (1979). Phys. Rev. B, 19, 3004-3021.

Nordberg, R., Brecht, H., Albridge, R. G., Fahlman, A. \& Van Wazer, J. R. (1970). Inorg. Chem. 9, 2469-2474.

Obrovac, M. N., Yuan G., Richard, M. N. \& Dahn, J. R. (1997). Appl. Phys. Lett. 71, 2262-2264.

Owens, A., Bayliss, S. C., Fraser, G. W. \& Gurman, S. J. (1997). Nucl. Instrum. Methods Phys. Res. A, 385, 556-558.

Schroeder, S., Moggridge, G., Lambert, R. \& Rayment, T. (1998). Spectroscopy for Surface Science, edited by R. Clark and R. Hester, Advances in Spectroscopy, Vol. 26. Chichester: Wiley.

Seah, M. P. \& Spencer, S. J. (2000). J. Electron Spectrosc. Relat. Phenom. 109, 291-308.

Stöhr, J. (1992). NEXAFS Spectroscopy, Springer Series in Surface Science, Vol. 25. New York: Springer.

Vlachos, D., Craven, A. J. \& McComb, D. W. (2001). J. Phys. Condens. Matter, 13 10799-10809. 